A\&A 385, 1049-1055 (2002)

DOI: $10.1051 / 0004-6361: 20020199$

(c) ESO 2002

\title{
Water maser emission in IC 342
}

\author{
A. Tarchi, C. Henkel, A. B. Peck, and K. M. Menten \\ Max-Planck-Institut für Radioastronomie, Auf dem Hügel 69, 53121 Bonn, Germany \\ Received 19 September 2001 / Accepted 4 February 2002

\begin{abstract}
The detection of $22 \mathrm{GHz}$ water vapor emission from IC 342 is reported, raising the detection rate among northern galaxies with IRAS point source fluxes $S_{100 \mu \mathrm{m}}>50 \mathrm{Jy}$ to $16 \%$. The maser, associated with a star forming region $\sim 10-15^{\prime \prime}$ west of the nucleus, consists of a single $0.5 \mathrm{~km} \mathrm{~s}^{-1}$ wide feature and reaches an isotropic luminosity of $10^{-2} L_{\odot}(D=1.8 \mathrm{Mpc})$. If the time variability is intrinsic, the maser size is $\lesssim 1.5 \times 10^{16} \mathrm{~cm}(\lesssim 0.5 \mathrm{mas})$ which corresponds to a brightness temperature of $\gtrsim 10^{9} \mathrm{~K}$. The linewidth, luminosity, and rapid variability are reminiscent of the $8 \mathrm{~km} \mathrm{~s}^{-1}$ super maser in Orion-KL. A velocity shift of $1 \mathrm{~km} \mathrm{~s}^{-1}$ within two weeks and subsequent rapid fading is explained in terms of a chance alignment of two dense molecular clouds. Observations at $22 \mathrm{GHz}$ toward Maffei 2 are also reported, yielding a $5 \sigma$ upper limit of $25 \mathrm{mJy}$ for a channel spacing of $1.05 \mathrm{~km} \mathrm{~s}^{-1}$.
\end{abstract}

Key words. galaxies: individual: IC 342, Maffei 2 - galaxies: starburst - ISM: molecules - masers radio lines: ISM

\section{Introduction}

Isotropic luminosities of extragalactic $22 \mathrm{GHz} \mathrm{H}_{2} \mathrm{O}$ masers span a huge range (see e.g. Ho et al. 1987; Koekemoer et al. 1995; Braatz et al. 1996), from $10^{-2}-10 L_{\odot}$ (the "kilomasers" consistent with the most intense masers seen in our Galaxy) to 10-500 $L_{\odot}$ (the "megamasers", found in LINERs and Seyfert 2s) and up to $6000 L_{\odot}$ (the "gigamaser" in TXS 2226-184). Interferometric observations, when available, show that the most luminous $\mathrm{H}_{2} \mathrm{O}$ sources arise from the nuclear region of their host galaxies, from circumnuclear tori, from entrained material near the surface of the nuclear jet, or by ambient gas amplifying the continuum emission of the jet. The weaker masers often mark locations of high mass star formation (for M 33, IC 10, and M 82, see e.g. Huchtmeier et al. 1978; Henkel et al. 1986; Baudry \& Brouillet 1996). The nature of the kilomasers in NGC 253 and M 51 (see Ho et al. 1987; Nakai \& Kasuga 1988) remained an enigma for more than a decade. Recently, however, it has been shown that the maser in M 51 is of nuclear origin (Hagiwara et al. 2001), suggesting that there might be a family of as yet unexplored "weak" $\mathrm{H}_{2} \mathrm{O}$ sources located near the nuclear engine of Seyfert 2 and LINER galaxies.

Extragalactic $\mathrm{H}_{2} \mathrm{O}$ masers are preferentially detected in nearby galaxies that are bright in the infrared (Braatz et al. 1997). While nuclear masers are of obvious interest, non-nuclear kilomasers are also important for a number of reasons: these sources allow us to pinpoint sites of massive

Send offprint requests to: A. Tarchi, e-mail: atarchi@mpifr-bonn.mpg.de star formation, to measure the velocity vectors of these regions through VLBI proper motion studies, and to determine true distances through complementary measurements of proper motion and radial velocity (e.g. Greenhill et al. 1993). We have therefore observed the nearby spiral galaxies IC 342 and Maffei 2, both of which exhibit prominent nuclear bars and strong molecular, infrared, and radio continuum emission (e.g. Hurt \& Turner 1991; Hurt et al. 1993; Turner \& Ho 1994; Henkel et al. 2000; Meier et al. 2000; Meier \& Turner 2001; Schulz et al. 2001). In the following we report the results of our observations.

\section{The observation and image processing}

Effelsberg The $6_{16}-5_{23}$ line of $\mathrm{H}_{2} \mathrm{O}$ (rest frequency: $22.23508 \mathrm{GHz}$ ) was observed with the $100-\mathrm{m}$ telescope of the MPIfR at Effelsberg ${ }^{1}$ toward IC 342 and Maffei 2. The full width to half power beamwidth was $40^{\prime \prime}$. A dual channel HEMT receiver provided system temperatures of $\sim 200 \mathrm{~K}$ on a main beam brightness temperature scale. The observations were carried out in a dual beam switching mode with a beam throw of $2^{\prime}$ and a switching frequency of $\sim 1 \mathrm{~Hz}$. Flux calibration was obtained by measurements of W3(OH) (for fluxes, see Mauersberger et al. 1988). Gain variations as a function of elevation were taken into account and the calibration error is expected to be no more than $\pm 10 \%$. The pointing accuracy was always better than $10^{\prime \prime}$

\footnotetext{
1 The 100-m telescope at Effelsberg is operated by the MaxPlanck-Institut für Radioastronomie (MPIfR) on behalf of the Max-Planck-Gesellschaft (MPG).
} 
VLA On May 12, 2001, IC 342 was observed with the Very Large Array $^{2}$ (VLA) in its B configuration. We observed with a single IF using full polarimetric information. The source $0538+498$ was used as flux calibrator. The nearby point source $0224+671$ was used for phase and bandpass calibration. "Referenced pointing" was performed during the observations (for details, refer to http://www. aoc.nrao.edu/vla/html/refpt.shtml).

The data were Fourier-transformed using natural weighting to produce a $2048 \times 2048 \times 103$ spectral line data cube, encompassing the central $100^{\prime \prime}$ of IC 342 . The 103 channels used, out of the 128 observed, cover a range in velocity of $\sim 8 \mathrm{~km} \mathrm{~s}^{-1}$ centered at $16 \mathrm{~km} \mathrm{~s}^{-1}$ LSR (the velocity of the line detected at Effelsberg). No continuum subtraction was needed. The data were deconvolved using the CLEAN algorithm (Högbom 1974). The restoring beam is $0^{\prime \prime} 4 \times 00^{\prime \prime} 3$ and the rms noise per channel is $\sim 10 \mathrm{mJy}$, consistent with the expected thermal noise.

\section{Results}

\subsection{Time variability}

Our single-dish observations towards Maffei 2 yielded no detection, with a $5 \sigma$ upper limit of $25 \mathrm{mJy}$ (channel spacing: $1.05 \mathrm{~km} \mathrm{~s}^{-1}$; velocity range: $-250 \mathrm{~km} \mathrm{~s}^{-1}<$ $V<+230 \mathrm{~km} \mathrm{~s}^{-1}$; epoch: Apr 3, 2001; position: $\alpha_{2000}=$ $\left.02^{\mathrm{h}} 41^{\mathrm{m}} 55^{\mathrm{s}} \cdot 2, \delta_{2000}=+59^{\circ} 36^{\prime} 11^{\prime \prime}\right)$.

On April 2, 2001, we obtained the first definite detection of water vapor emission in IC $342^{3}$ (see also Sect. 4.1). During the next night the detection was confirmed with a velocity resolution sufficient to resolve the line profile (Fig. 1a). The detected feature lies at $V_{\mathrm{LSR}}=$ $16 \mathrm{~km} \mathrm{~s}^{-1}$ and has a linewidth of $\sim 0.5 \mathrm{~km} \mathrm{~s}^{-1}$; on April 2, no other component was seen at velocities $-175 \mathrm{~km} \mathrm{~s}^{-1}<$ $V<+310 \mathrm{~km} \mathrm{~s}^{-1}$ (channel spacing: $1.05 \mathrm{~km} \mathrm{~s}^{-1} ; 5 \sigma$ noise level: $16 \mathrm{mJy})$. A high line intensity ( 100 mJy) and good weather conditions allowed us to map the emitting region. Figure 2 shows the spectra taken towards six positions near the center of the galaxy. On April 22, observations were performed towards the offset position $\left(-10^{\prime \prime}, 0^{\prime \prime}\right)$, close to the peak position inferred by the map. The flux density had almost doubled (compare Figs. 1a and b) which is more than the expected $20 \%$ increase for a point source located $\sim 10^{\prime \prime}$ off our previously observed $\left(0^{\prime \prime}, 0^{\prime \prime}\right)$ position. Further measurements were performed on May 7 (Fig. 1c). The peak and integrated intensities

\footnotetext{
2 The National Radio Astronomy Observatory is a facility of the National Science Foundation operated under cooperative agreement by Associated Universities, Inc.

3 Water masers in IC 342 were previously reported by Huchtmeier et al. (1978) well outside the nuclear region. However, their spectrum from IC 342-4 was not shown and its velocity $\left(-54 \mathrm{~km} \mathrm{~s}^{-1}\right)$ is highly "forbidden" (there is no $\mathrm{H} \mathrm{I}$ at this velocity; see e.g. Crosthwaite et al. 2000), while the spectral profile from IC $342-3$, peaking at $-52 \mathrm{~km} \mathrm{~s}^{-1}$, looks tentative. If the feature is real, an association with the galactic Perseus arm cannot be excluded.
}

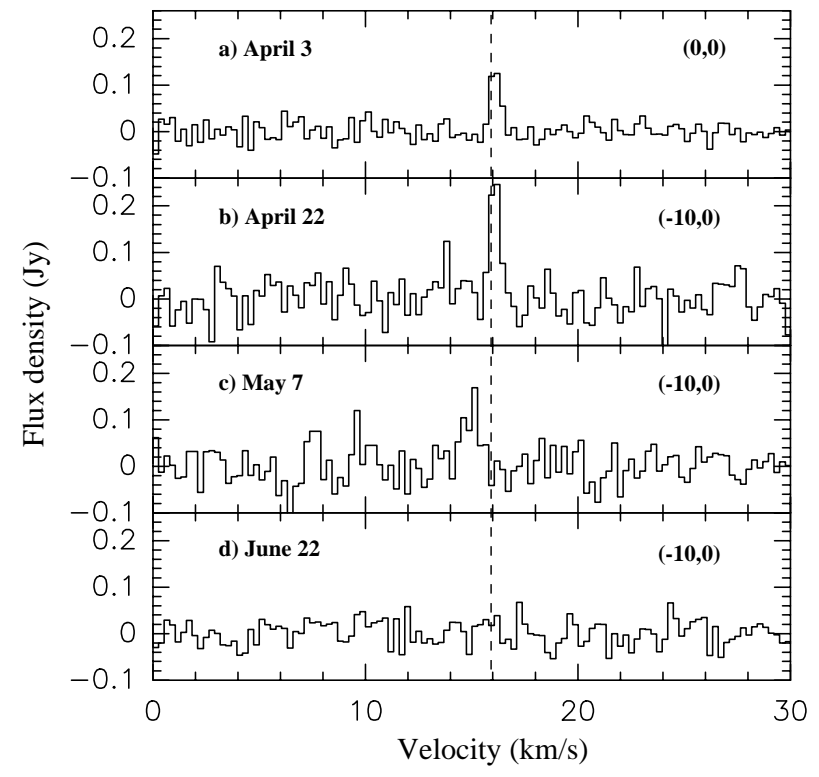

Fig. 1. The $\mathrm{H}_{2} \mathrm{O}$ maser feature observed with high velocity resolution (channel spacings after smoothing four contiguous channels are $0.26 \mathrm{~km} \mathrm{~s}^{-1}$ ) on a) April 3, b) April 22, c) May 7, and d) June 22. The first spectrum has been taken at the $\left(0^{\prime \prime}\right.$, $\left.0^{\prime \prime}\right)$ position, the others at $\left(-10^{\prime \prime}, 0^{\prime \prime}\right)$ relative to the position given in footnote "a" of Table 1 . The dashed line indicates $V_{\mathrm{LSR}}=16 \mathrm{~km} \mathrm{~s}^{-1}$.

are smaller and the velocity of the line is blue-shifted by $\sim 1 \mathrm{~km} \mathrm{~s}^{-1}$. Unfortunately, no emission above $\sim 30 \mathrm{mJy}(3 \sigma$ level; $0.08 \mathrm{~km} \mathrm{~s}^{-1}$ channel spacing) was detected in the $22 \mathrm{GHz}$ VLA B-array data taken on May 12. This fading by at least a factor of 3 within 5 days implies a size scale of $\lesssim 1.5 \times 10^{16} \mathrm{~cm}(\$ 900 \mathrm{AU})$ or $\lesssim 0.5$ mas at a distance of $1.8 \mathrm{Mpc}$ (McCall et al. 1989; see also Sect. 4.3); the corresponding brightness temperature is $z 10^{9} \mathrm{~K}$. The most recent spectrum was obtained on June 22 at Effelsberg (Fig. 1d). Confirming the VLA result, no maser signal was seen above $30 \mathrm{mJy}$ ( $3 \sigma$; channel spacing: $1.05 \mathrm{~km} \mathrm{~s}^{-1}$ ).

Offset positions, on-source integration times, Gaussian fit parameters, and $\mathrm{H}_{2} \mathrm{O}$ luminosities assuming isotropic emission (see e.g. Henkel et al. 1998) are given in Table 3.1.

\subsection{Maser position}

Fitting a synthetic Gaussian to the data taken on April 3 (see Table 1 and Fig. 2), i.e. minimizing the sum of the difference squared between calculated and observed peak and integrated flux densities, we can obtain an accurate position of the emitting region: $\alpha_{2000}=03^{\mathrm{h}} 46^{\mathrm{m}} 46^{\mathrm{s}} 3, \delta_{2000}=$ $+68^{\circ} 05^{\prime} 46^{\prime \prime}$. This is $13^{\prime \prime}$ to the west of our center position (see Table 1) that coincides with the optical nucleus and the $2 \mu \mathrm{m}$ peak (van der Kruit 1973; Becklin et al. 1980). The accuracy of the derived maser position is affected by the following uncertainties:

Relative pointing accuracy: on April 3, the average pointing correction was $\sigma_{\text {rpoi }} \sim 2^{\prime \prime}$. Using the nearby ultracompact $\mathrm{H}$ II region $\mathrm{W} 3(\mathrm{OH})$ as pointing source slightly degraded this pointing accuracy because of the presence of 


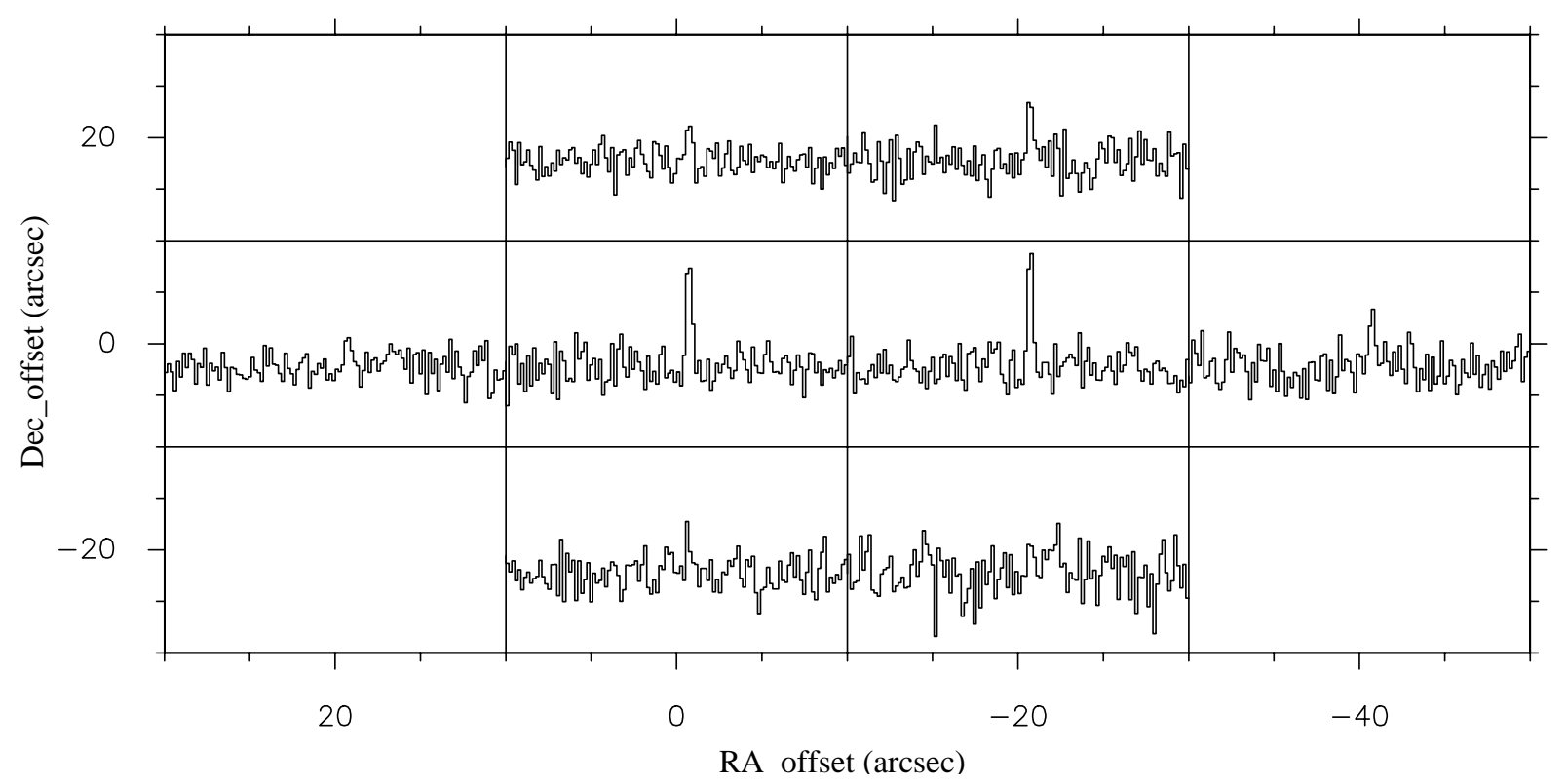

Fig. 2. $\mathrm{H}_{2} \mathrm{O}$ spectra obtained toward the central region of IC 342 . Positions are offsets relative to $\alpha_{2000}=03^{\mathrm{h}} 46^{\mathrm{m}} 48^{\mathrm{s}} \cdot 6$ and $\delta_{2000}=+68^{\circ} 05^{\prime} 46^{\prime \prime}$. Note that the spacing between two individual spectra $\left(20^{\prime \prime}\right)$ is approximately half of the size of the $100-\mathrm{m}$ Effelsberg telescope beam at $22 \mathrm{GHz}\left(40^{\prime \prime}\right)$. Averaging four contiguous channels, the spectra have been smoothed to a spacing of $0.26 \mathrm{~km} \mathrm{~s}^{-1}$.

a strong $\mathrm{H}_{2} \mathrm{O}$ maser $6^{\prime \prime}$ to the west $\left(\mathrm{W} 3\left(\mathrm{H}_{2} \mathrm{O}\right)\right.$; e.g. Reid et al. 1995). The error introduced by the non-negligible flux contribution of the maser is $\sigma_{\mathrm{W} 3(\mathrm{OH})}=1$ '. 2 .

Absolute pointing accuracy: when moving the Effelsberg antenna from W3(OH) to IC 342, there may be an additional pointing offset caused by the change in azimuth and elevation. On April 3, relatively large telescope movements led to a pointing shift of $2^{\prime \prime}$ in azimuth and $4^{\prime \prime}$ in elevation. Considering the improvement in weather conditions when subsequently mapping IC 342, and accounting for the much smaller distance between target (IC 342) and pointing source (W3(OH)), we estimate the absolute pointing error to be $\sigma_{\text {apoi }}=2^{\prime \prime} .5$.

Spectral noise: for data taken during a single night, relative calibration should be excellent. The relevant factor affecting line intensity ratios is thus noise. Assuming a Gaussian beamshape (beamwidth: $40^{\prime \prime}$ ), resulting peak and integrated $\mathrm{H}_{2} \mathrm{O}$ flux densities were calculated for various maser positions. Agreement with the observed intensity limit at the $\left(-20^{\prime \prime},-20^{\prime \prime}\right)$ position was taken as an additional check. The resulting positional error is $\sigma_{\text {map }}=4^{\prime \prime}$.

The assumption that all errors can be statistically added yields a total positional uncertainty of $\sigma_{\text {tot }}=\sqrt{\sigma_{\text {rpoi }}^{2}+\sigma_{\text {apoi }}^{2}+\sigma_{\mathrm{W} 3(\mathrm{OH})}^{2}+\sigma_{\text {map }}^{2}} \sim 5^{\prime \prime}$.

\section{Discussion}

\subsection{Galactic versus extragalactic $\mathrm{H}_{2} \mathrm{O}$ emission}

IC 342 is located at a galactic longitude of $138^{\circ}$ and latitude $10^{\circ}$, i.e. behind the northern outskirts of the Perseus arm, the Cam OB1 association, and a Local Arm of the Milky Way (e.g. Digel et al. 1996; Dame et al. 2001). Local
Standard of Rest (LSR) velocities of the Perseus arm range from -60 to $-30 \mathrm{~km} \mathrm{~s}^{-1}$, consistent with the maser features reported by Huchtmeier et al. (1978), but significantly removed from the radial velocity of the maser reported here. For the Cam OB1 association and the Local Arm, Digel et al. (1996) find velocities up to -5 and $+7 \mathrm{~km} \mathrm{~s}^{-1}$, still below the measured maser velocity. Furthermore, only a few of the hundreds of known galactic $\mathrm{H}_{2} \mathrm{O}$ masers have been detected at $b^{\mathrm{II}} \sim 10^{\circ}$ (e.g. Valdettaro et al. 2001). All of these arguments support an extragalactic origin of the maser. In addition, the IC 342 isovelocity contour at $V_{\mathrm{LSR}} \sim 16 \mathrm{~km} \mathrm{~s}^{-1}$, obtained from $\mathrm{CO}$ interferometric data, is consistent with a maser location $10-15^{\prime \prime}$ west of its nucleus (e.g. Lo et al. 1984; Sakamoto et al. 1999). This agrees with the position determined in Sect. 3.2. We therefore conclude that the $\mathrm{H}_{2} \mathrm{O}$ source is associated with the central region of IC 342 (for an illustration, see Fig. 3).

\subsection{Search for an optical counterpart}

Arising from the central region of IC 342 , but being displaced from the nucleus, the $\mathrm{H}_{2} \mathrm{O}$ maser is likely associated with a prominent star forming region.

Figure 4 shows an optical B-band image of the central region of IC 342, taken from the $\mathrm{XDSS}^{4}$. The maser emitting region (white circle) coincides with an arc-like

\footnotetext{
${ }^{4}$ The Digitized Sky Surveys were produced at the Space Telescope Institute under U.S. Government grant NAGW2166. The images of these surveys are based on photographic data obtained using the Oschin Schmidt Telescope on Palomar Mountain and the UK Schmidt Telescope. The plates were processed into the present compressed digital form with the permission of these institutions.
} 
Table 1. $\mathrm{H}_{2} \mathrm{O}$ in IC 342: observations details and line parameters.

\begin{tabular}{|c|c|c|c|c|c|c|c|c|c|}
\hline Obs. Date & $\begin{array}{c}(\Delta \alpha, \Delta \delta)^{\mathrm{a})} \\
\left({ }^{\prime \prime},{ }^{\prime \prime}\right)\end{array}$ & Tel. & $\begin{array}{c}t_{\mathrm{obs}}^{\mathrm{b})} \\
(\mathrm{min})\end{array}$ & $\begin{array}{c}B W \\
(\mathrm{MHz})\end{array}$ & $\begin{array}{l}\text { Vel. Res. } \\
\left(\mathrm{km} \mathrm{s}^{-1}\right)\end{array}$ & $\begin{array}{c}S_{\text {peak }}^{\mathrm{c})} \\
(\mathrm{Jy})\end{array}$ & $\begin{array}{l}V_{\mathrm{LSR}}^{\mathrm{d})}(\mathrm{kms} \\
\quad\end{array}$ & $\begin{array}{l}\Delta V_{1 / 2} \\
\left.\mathrm{~S}^{-1}\right)\end{array}$ & $\begin{array}{c}\text { Luminosity }^{\mathrm{e})} \\
\left(L_{\odot}\right)\end{array}$ \\
\hline 02 Apr. $2001^{f)}$ & $(0,0)$ & $\mathrm{EFF}$ & 180 & 40 & 1.05 & - & $16.16(0.03)$ & - & $(8.3 \pm 0.6) \times 10^{-3}$ \\
\hline 03 Apr. 2001 & $(-40,0)$ & $\mathrm{EFF}$ & 30 & 20 & 0.07 & $0.07 \pm 0.03$ & $16.15(0.09)$ & $0.61(0.28)$ & $(3.2 \pm 1.0) \times 10^{-3}$ \\
\hline 03 Apr. 2001 & $(-20,-20)$ & $\mathrm{EFF}$ & 20 & $"$ & $"$ & $<0.07$ & & & $<3 \times 10^{-3}$ \\
\hline 03 Apr. 2001 & $(-20,0)$ & $\mathrm{EFF}$ & 30 & " & $"$ & $0.18 \pm 0.03$ & $16.08(0.03)$ & $0.49(0.06)$ & $(6.5 \pm 0.7) \times 10^{-3}$ \\
\hline 03 Apr. 2001 & $(-20,20)$ & $\mathrm{EFF}$ & 30 & $"$ & $"$ & $0.10 \pm 0.03$ & $16.07(0.06)$ & $0.53(0.13)$ & $(3.9 \pm 0.8) \times 10^{-3}$ \\
\hline 03 Apr. 2001 & $(0,-20)$ & $\mathrm{EFF}$ & 30 & $"$ & $"$ & $0.06 \pm 0.02$ & $16.06(0.08)$ & $0.46(0.14)$ & $(2.0 \pm 0.6) \times 10^{-3}$ \\
\hline 03 Apr. 2001 & $(0,0)$ & $\mathrm{EFF}$ & 30 & $"$ & $"$ & $0.17 \pm 0.02$ & $16.14(0.02)$ & $0.58(0.05)$ & $(7.3 \pm 0.6) \times 10^{-3}$ \\
\hline 03 Apr. 2001 & $(0,20)$ & $\mathrm{EFF}$ & 30 & " & $"$ & $0.06 \pm 0.04$ & $16.15(0.06)$ & $0.40(0.20)$ & $(1.8 \pm 0.6) \times 10^{-3}$ \\
\hline 03 Apr. 2001 & $(20,0)$ & $\mathrm{EFF}$ & 30 & $"$ & . & $0.04 \pm 0.02$ & $16.14(0.13)$ & $0.64(0.22)$ & $(1.9 \pm 0.7) \times 10^{-3}$ \\
\hline 22 Apr. 2001 & $(-10,0)$ & $\mathrm{EFF}$ & 8 & $"$ & $"$ & $0.30 \pm 0.06$ & $16.11(0.04)$ & $0.54(0.08)$ & $(12.0 \pm 1.5) \times 10^{-3}$ \\
\hline 07 May 2001 & $(-10,0)$ & $\mathrm{EFF}$ & 15 & $"$ & $"$ & $0.17 \pm 0.05$ & $14.98(0.08)$ & $0.81(0.18)$ & $(10.3 \pm 1.9) \times 10^{-3}$ \\
\hline 12 May 2001 & & VLA & 120 & 0.781 & 0.08 & $<0.03$ & & & $<1.2 \times 10^{-3}$ \\
\hline 22 Jun. 2001 & $(-10,0)+(0,0)$ & $\mathrm{EFF}$ & 35 & 40 & 1.05 & $<0.03$ & & & $<1.7 \times 10^{-3}$ \\
\hline
\end{tabular}

a) The position offset is relative to $\alpha_{2000}=03^{\mathrm{h}} 46^{\mathrm{m}} 48^{\mathrm{s}} \cdot 6$ and $\delta_{2000}=+68^{\circ} 05^{\prime} 46^{\prime \prime}$.

b) $t_{\text {obs }}$ (Effelsberg $22 \mathrm{GHz} \mathrm{H} \mathrm{H}_{2} \mathrm{O}$ integration time) includes time for on- and off-source integration.

c) Peak fluxes calculated from integrated line intensities divided by linewidths (both obtained from Gaussian fits using the data reduction software package "CLASS"); if no signal is detected, $3 \sigma$ limits are given for a channel spacing of $0.53 \mathrm{~km} \mathrm{~s}$ (Apr. 3 ), $0.08 \mathrm{~km} \mathrm{~s}^{-1}$ (May 12), and $1.05 \mathrm{~km} \mathrm{~s}^{-1}$ (June 22). Since the data taken on April 3 were used for the computation of the position of the maser, where only (presumably negligible) relative calibration errors are relevant, stated errors do not include absolute calibration errors ( $\pm 10 \%$; see Sect. 2$)$.

d) $\mathrm{LSR}=$ Local Standard of Rest.

e) Obtained via $\left[L_{\mathrm{H}_{2} \mathrm{O}} / L_{\odot}\right]=0.023 \times\left[\int S \mathrm{~d} V / \mathrm{Jy} \mathrm{km} \mathrm{s}^{-1}\right] \times[D / \mathrm{Mpc}]^{2}, D=1.8 \mathrm{Mpc}$. Corresponding luminosity limits refer to $\Delta V_{1 / 2}=0.55 \mathrm{~km} \mathrm{~s}^{-1}$, the approximate width of the $\mathrm{H}_{2} \mathrm{O}$ feature. Errors do not include the estimated calibration uncertainty of $\pm 10 \%$ (see Sect. 2 and footnote c).

f) Velocity resolution too coarse to determine meaningful values for the peak flux density and linewidth.

structure extending E-S and is associated with a chain of sources that appear to be HII regions. The presence of optically bright regions, likely sites of star formation activity, close to the maser position is confirmed by an $\mathrm{H} \alpha$ $\left(\lambda 6563 \AA\right.$ ) HST WFPC2 picture $^{5}$ (see also Meier et al. 2000).

\subsection{A comparison with other maser sources}

Although seen almost face-on (inclination $i \sim 25^{\circ}$; e.g. Sage \& Solomon 1991), IC 342 is believed to resemble the Milky Way in many ways. At the commonly assumed $D \sim$ $2 \mathrm{Mpc}$ (but see Krismer et al. 1995 for a larger distance), the giant molecular clouds near the center of IC 342 have similar linear sizes to the Sgr A and Sgr B2 clouds in our Galaxy. The inner 400 pc of IC 342 and the Galaxy have almost the same far infrared luminosity and the $2 \mu \mathrm{m}$ luminosities indicate similar stellar masses (Downes et al. 1992).

\footnotetext{
${ }^{5}$ Some of the data mentioned in this paper were obtained from the Multimission Archive at the Space Telescope Institute (MAST). STScI is operated by the Association of Universities for Research in Astronomy, Inc. under NASA contract NAS 5-26555. Support for MAST for non-HST data is provided by the NASA Office of Space Science via grant NAG 5-7584 and by other grants and contracts.
}

Does this similarity extend to the $22 \mathrm{GHz}$ maser emission? While there are a number of $\mathrm{H}_{2} \mathrm{O}$ maser sources in the nuclear region of the Milky Way (Güsten \& Downes 1983; Taylor et al. 1993; Yusef-Zadeh \& Mehringer 1995), the emission is dominated by Sgr B2 (e.g. Waak \& Mayer 1974; Morris 1976; Genzel et al. 1976; Elmegreen et al. 1980; Kobayashi et al. 1989). Like the $\mathrm{H}_{2} \mathrm{O}$ maser in IC 342, Sgr B2 is displaced from the galactic nucleus. The integrated luminosity of its numerous spatial components is $L_{\mathrm{H}_{2} \mathrm{O}} \sim 5 \times 10^{-3} L_{\odot}$, again not inconsistent with the data from IC 342. The lineshapes are, however, quite different: While Sgr B2 is characterized by a variety of narrow components that have flux densities of a few hundred Jy, the velocity component we have detected in IC 342 is stronger than any related feature by at least an order of magnitude.

Occasionally, prominent galactic star forming regions show narrow $\left(\sim 0.5 \mathrm{~km} \mathrm{~s}^{-1}\right)$ flaring components that are exceptionally bright. Such flares were observed in W 31 A and W 49 (Liljeström et al. 1989; Lekht et al. 1995). The prototype of these flares is the $8 \mathrm{~km} \mathrm{~s}^{-1}$ super maser in Orion-KL (e.g. Garay et al. 1989). During several months, the narrow highly linearly polarized maser reached flux densities in excess of 5 MJy. Surpassing the flux of any other velocity component by more than an order of magnitude and reaching a peak luminosity of $L_{\mathrm{H}_{2} \mathrm{O}} \sim$ $10^{-2} L_{\odot}$, the feature seems to be similar to that seen 


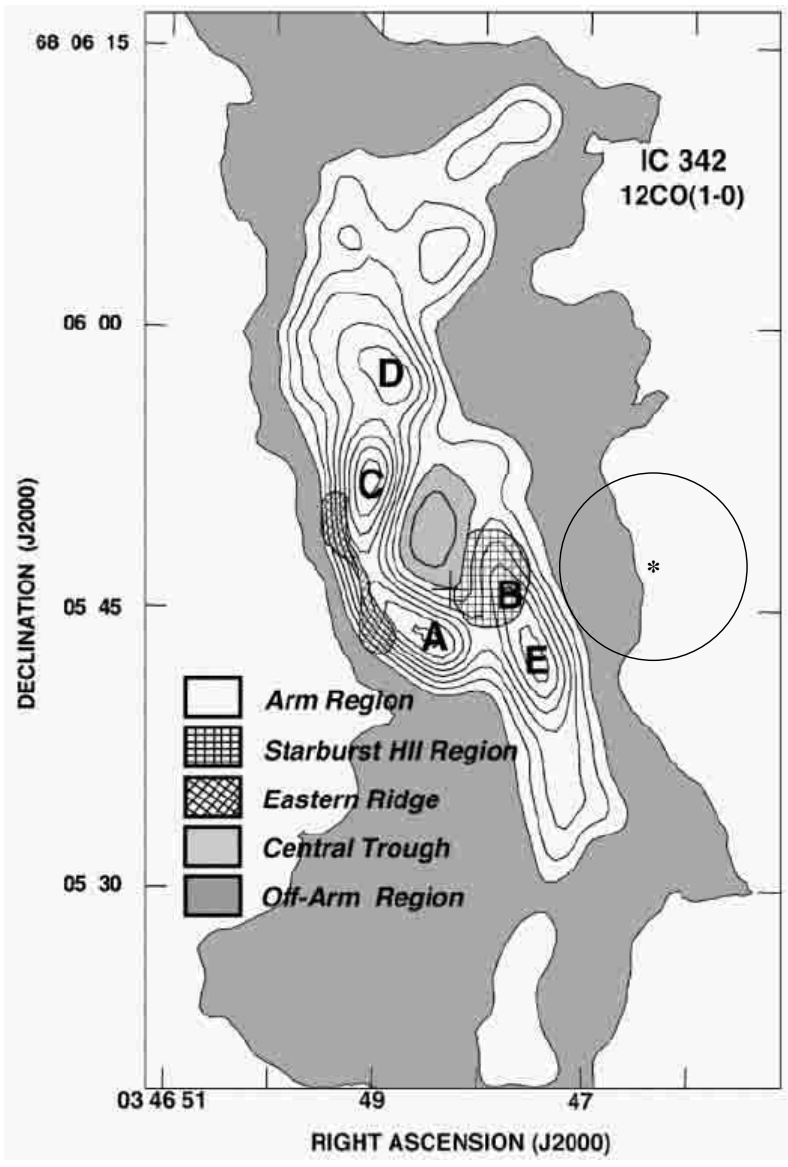

Fig. 3. A map of integrated ${ }^{12} \mathrm{CO} J=1-0$ emission from the central region of IC 342 (Meier et al. 2000). The asterisk indicates the location of the water maser with the circle marking the positional uncertainty (Sect. 3.2). The letters label the five giant molecular clouds identified by Downes et al. (1992).

in IC 342. There is another even more impressive narrow flaring maser, which dwarfed neighboring features: the $-324 \mathrm{~km} \mathrm{~s}^{-1}$ component in the nearby irregular galaxy IC 10 (Becker et al. 1993; Argon et al. 1994; Baan \& Haschick 1994). This prominent flare, reaching a luminosity of $\sim 1 L_{\odot}(\sim 100 \mathrm{Jy} ; D \sim 0.8 \mathrm{Mpc})$, lasted for a few years. As unpublished spectra from Effelsberg show, the lineshape of the maser also resembles that of the flaring feature in Orion-KL.

While it is not farfetched to assume that the flares in IC 342, IC 10, and Orion-KL are caused by the same physical phenomenon (but see Argon et al. 1994), the actual situation remains unclear. For Orion-KL, observational constraints are quite strict. There is no significant background continuum source and the high intensity seems to suggest saturated emission and a collisional pump (e.g. Garay et al. 1989). The small linewidth, however, appears to be incompatible with such a scenario (Nedoluha \& Watson 1991). Solving this apparent inconsistency requires either very small beaming angles, which are readily obtained by a chance alignment of two masing clouds (e.g. Deguchi \& Watson 1989; Elitzur et al. 1991), or the inclusion of efficient photon scattering, which can account for linewidths $\sim 0.5 \mathrm{~km} \mathrm{~s}^{-1}$ even in the case of saturation (e.g. Elitzur 1990).

While luminosity, linewidth, and flux variations are reminiscent of the $8 \mathrm{~km} \mathrm{~s}^{-1}$ super maser in Orion-KL, our $\mathrm{H}_{2} \mathrm{O}$ profiles show a significant velocity shift between April 22 and May 7 (Figs. 1b and c) that has not been seen in the Orion-KL flaring component. The shift, $\sim 1 \mathrm{~km} \mathrm{~s}^{-1}$ (Table 1; see also Sect. 3.1), is too large to be explained by a change in the relative intensities of the three main hyperfine components (see e.g. Fiebig \& Güsten 1989). A change with time in the peak velocity could originate from a variation in the relative intensity of different emitting regions leading to the swapping-over of velocity peaks (see e.g. Wu et al. 1999). Such intensity fluctuations have been found to be sometimes "correlated" with observed time scales ranging from some days up to weeks (intrinsic time scales are more difficult to deduce because they strongly depend on the degree of saturation of the masers; see e.g. Genzel \& Downes 1977; Rowland \& Cohen 1986). Time scales equal or greater than some months are also predicted for maser flux density variations (distinct from flares) due to refractive interstellar scintillations (Simonetti et al. 1993). However, a simpler model for explaining both the flare and the shift is, in our opinion, that the latter has a kinematic origin. Adopting the previously outlined scenario of a chance alignment of two masing clouds along the line-ofsight, motion of the foreground relative to the background cloud along the plane of the sky and velocity structure in the foreground cloud could explain the observed data. A velocity gradient in the foreground cloud would then first shift the line velocity; once velocities are reached that are not matched within the background cloud, the flux density of the maser rapidly drops. Assuming that the distance between these clouds is $\ll 1.8 \mathrm{Mpc}$ and that their relative velocity in the plane of the sky is at the order of $100 \mathrm{~km} \mathrm{~s}^{-1}$, this implies a cloud velocity gradient of up to $1 \mathrm{~km} \mathrm{~s}^{-1} / \mathrm{AU}$ during the time the source was monitored.

An almost identical scenario was proposed by Boboltz et al. (1998) to account for the velocity shift of the flaring water maser component at $-66 \mathrm{~km} \mathrm{~s}^{-1}$ in W49N. The similarity in velocity shift $\left(\sim 0.5 \mathrm{~km} \mathrm{~s}^{-1}\right)$ and time scale (58 days) of the event in W49N w.r.t the one in IC 342 , indicates a common origin.

\subsection{Extragalactic maser detection rates}

While typical searches for extragalactic maser sources have only yielded detection rates between zero (e.g. Henkel et al. 1998) and a few percent (e.g. Henkel et al. 1984; Braatz et al. 1996), there exists one sample with detection rates $>10 \%$ : these are the northern $\left(\delta>-30^{\circ}\right)$ extragalactic IRAS (Infrared Astronomy Satellite) point sources with $100 \mu \mathrm{m}$ fluxes in excess of $50 \mathrm{Jy}$ (for a source list, see Henkel et al. 1986; Maffei 2 with $S_{100 \mu \mathrm{m}} \sim 200$ Jy should be added to the list). There is a total of 44 galaxies, two ultraluminous galaxies at intermediate distances (NGC 3690 and Arp 220) and 42 nearby sources $\left(V<3000 \mathrm{~km} \mathrm{~s}^{-1}\right)$. 


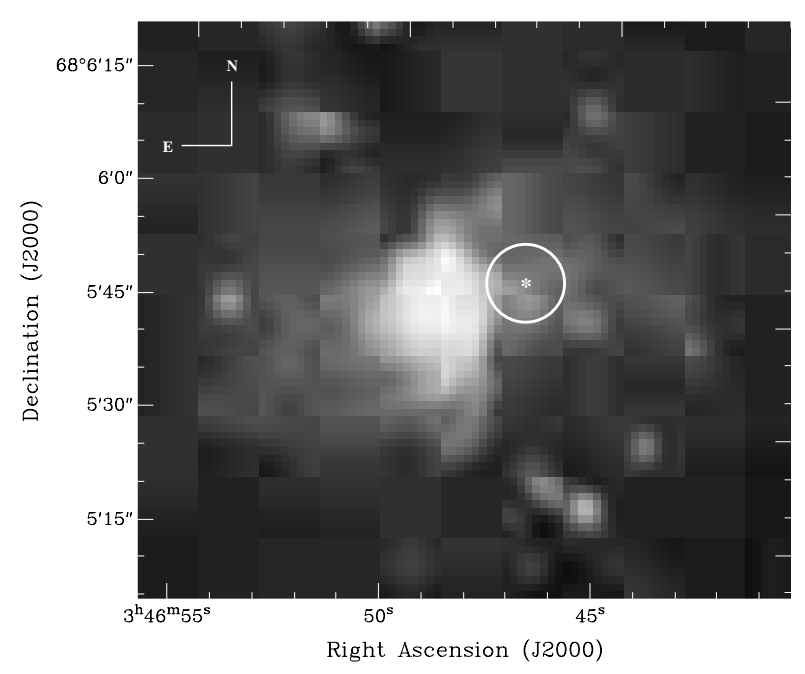

Fig. 4. XDSS B-band image of the central region of IC 342 . The asterisk indicates the water maser emitting position. The radius of the circle indicates its positional error as outlined in Sect. 3.2.

Among these, seven (16\%) are known to contain $\mathrm{H}_{2} \mathrm{O}$ masers in their nuclear region. Two of these contain megamasers (NGC 1068 and NGC 3079), two are possibly nuclear kilomasers (NGC 253 and M 51; for details, see Sect. 1), and three are associated with prominent sites of star formation (IC 10, IC 342, M 82). Among the subsample of 19 sources with $100 \mu \mathrm{m}$ fluxes in excess of $100 \mathrm{Jy}$, five were so far detected in $\mathrm{H}_{2} \mathrm{O}$, yielding a detection rate in excess of $20 \%$. Since few deep integrations have been obtained toward these sources, more $\mathrm{H}_{2} \mathrm{O}$ detections can be expected from this promising sample in the near future.

\section{Conclusions}

Having detected water vapor emission in the galaxy IC 342, we have obtained the following main results:

1. The maser arises from a location $10-15^{\prime \prime}$ to the west of the center of the galaxy. It is thus a maser associated with a powerful star forming region at a projected distance of $\sim 100 \mathrm{pc}(D=1.8 \mathrm{Mpc})$ from the nucleus.

2. Luminosity $\left(10^{-2} L_{\odot}\right)$, linewidth $\left(0.5 \mathrm{~km} \mathrm{~s}^{-1}\right)$, and rapid flux density variations are reminiscent of the flaring $8 \mathrm{~km} \mathrm{~s}^{-1}$ super maser feature in Orion-KL. It seems that we have observed an exceptional outburst of a maser that is usually well below the detection threshhold.

3. Time variability, if intrinsic, yields a maser size of $\$ 1.5 \times 10^{16} \mathrm{~cm}^{-3}$ ( $\left.\$ 0.5 \mathrm{mas}\right)$, and a brightness temperature of $\gtrsim 10^{9} \mathrm{~K}$.

4. A velocity shift of $1 \mathrm{~km} \mathrm{~s}^{-1}$ within 16 days and a subsequent decrease in flux density by at least a factor of three within 5 days can be explained by a chance alignment of two dense molecular clouds along the lineof-sight, both of them associated with IC 342 .

5. Among galaxies with IRAS point source flux densities of $S_{100 \mu \mathrm{m}}>50 \mathrm{Jy}, 16 \%$ are now known to contain masers in their inner regions. Since deep $\mathrm{H}_{2} \mathrm{O}$ integrations have been obtained towards only a few of them, more detections from this sample can be expected in the near future.

Acknowledgements. We wish to thank P. Diamond for critically reading the manuscript and Nikolaus Neininger for useful discussion. We are also endebted to the operators at the $100-\mathrm{m}$ telescope, and to Michael Rupen and the NRAO analysts, for their cheerful assistance with the observations.

\section{References}

Argon, A. L., Greenhill, L. J., Moran, J. M., et al. 1994, ApJ, 422,586

Baan, W. A., \& Haschick, A. D. 1994, ApJ, 424, L33

Baudry, A., \& Brouillet, N. 1996, A\&A, 316, 188

Becker, R., Henkel, C., Wilson, T. L., \& Wouterloot, J. G. A. 1993, A\&A, 268, 483

Becklin, E. E., Gatley, I., Matthews, K., et al. 1980, ApJ, 236, 441

Boboltz, D. A., Simonetti, J. H., Dennison, B., et al. 1998, ApJ, 509, 256

Braatz, J. A., Wilson, A. S., \& Henkel, C. 1996, ApJS, 106, 51

Braatz, J. A., Wilson, A. S., \& Henkel, C. 1997, ApJS, 110, 321

Crosthwaite, L. P., Turner, J. L., \& Ho, P. T. P. 2000, AJ, 119, 1720

Dame, T. M., Hartmann, D., \& Thaddeus, P. 2001, ApJ, 547, 792

Deguchi, S., \& Watson, W. D. 1989, ApJ, 340, L17

Digel, S. W., Lyder, D. A., Philbrick, A. J., Puche, D., \& Thaddeus, P. 1996, ApJ, 458, 561

Downes, D., Radford, S. J. E., Guilloteau, S., et al. 1992, A\&A, 262, 424

Elitzur, M. 1990, ApJ, 350, L7

Elitzur, M., McKee, C. F., \& Hollenbach, D. J. 1991, ApJ, 367, 333

Elmegreen, B. G., Genzel, R., Moran, J. M., Reid, M. J., \& Walker, R. C. 1980, ApJ, 241, 1007

Fiebig, D., \& Güsten, R. 1989, A\&A, 214, 333

Garay, G., Moran, J. M., \& Haschick, A. D. 1989, ApJ, 338, 244

Genzel, R., \& Downes, D. 1977, A\&AS, 30, 145

Genzel, R., Downes, D., \& Bieging, J. 1976, MNRAS, 177, 101

Greenhill, L. J., Moran, J. M., Reid, M. J., Menten, K. M., \& Hirabatashi, H. 1993, ApJ, 406, 482

Güsten, R., \& Downes, D. 1983, A\&A, 117, 343

Hagiwara, Y., Henkel, C., Menten, K., \& Nakai, N. 2001, ApJ, 560, L37

Henkel, C., Güsten, R., Downes, D., et al. 1984, A\&A, 141, L1

Henkel, C., Wouterloot, J. G. A., \& Bally, J. 1986, A\&A, 155, 193

Henkel, C., Wang, Y. P., Falcke, H., Wilson, A. S., \& Braatz, J. A. 1998, A\&A, 335, 463

Henkel, C., Mauersberger, R., Peck, A. B., Falcke, H., \& Hagiwara, Y. 2000, A\&A, 361, L45

Ho, P. T. P., Martin, R. N., \& Henkel, C. 1987, ApJ, 320, 663

Högbom, J. A. 1974, A\&AS, 15, 417

Huchtmeier, W., Witzel, A., Kühr, H., Pauliniy-Toth, I. I., \& Roland, J. 1978, A\&A, 64, L21

Hurt, R. L., \& Turner, J. L. 1991, ApJ, 377, 434 
Hurt, R. L., Turner, J. L., Ho, P. T. P., \& Martin, R. N. 1993, ApJ, 404, 602

Kobayashi, H., Ishiguro, M., Chikada, Y., et al. 1989, PASJ, 41, 141

Koekemoer, A. M., Henkel, C., Greenhill, L. J., Dey, A., van Breugel, W., et al. 1995, Nature, 378, 697

Krismer, M., Tully, R. B., \& Gioa, M. 1995, AJ, 110, 1584

Lekht, E. E., Mendoza-Torres, E., \& Sorochenko, R. L. 1995, ApJ, 443, 222

Liljeström, T., Mattila, K., Toriseva, M., \& Anttila, R. 1989, A\&AS, 79, 19

Lo, K.-Y., Berge, G. L., Claussen, M. J., Heiligman, G. M., Leighton, R. B., et al. 1984, ApJ, 282, L59

Mauersberger, R., Wilson, T. L., \& Henkel, C. 1988, A\&A, 201, 123

McCall, M. L. 1989, AJ, 97, 1341

Meier, D. S., Turner, J. L., \& Hurt, R. L. 2000, ApJ, 531, 200

Meier, D. S., \& Turner, J. L. 2001, ApJ, 551, 687

Morris, M. 1976, ApJ, 210, 100

Nakai, N., \& Kasuga, T. 1988, PASJ, 40, 139

Nedoluha, G. E., \& Watson, W. D. 1991, ApJ, 367, L63
Reid, M. J., Argon, A. L., Masson, C. R., et al. 1995, ApJ, 443, 238

Rowland, P. R., \& Cohen, R. J. 1986, MNRAS, 220, 233

Sage, L. J., \& Solomon, P. M. 1991, ApJ, 380, 392

Sakamoto, K., Okumura, S. K., Ishizuki, S., \& Scoville, N. Z. 1999, ApJS, 124, 403

Schulz, A., Güsten, R., Köster, B., \& Krause, D. 2001, A\&A, 371,25

Simonetti, J. H., Diamond, P. J., Uphoff, J. A., Boboltz, D., \& Dennison, B. 1993, in Astrophysical Masers, ed. A. W. Clegg, \& G. E. Nedoluha (Springer Verlag, Berlin), Lecture Notes in Phys., 412,311

Taylor, G. B., Morris, M., \& Schulman, E. 1993, AJ, 106, 1978

Turner, J. L., \& Ho, P. T. P. 1994, ApJ, 421, 122

Valdettaro, R., Palla, F., Brand, J., et al. 2001, A\&A, 368, 845 van der Kruit, P. C. 1973, A\&A, 29, 249

Waak, J. A., \& Mayer, J. A. 1974, ApJ, 189, 67

Wu, Y.-F., Shi, J.-R., Wang, J.-Z., Jiang, D.-R., \& Huang, X.-Y. 1999, ChA\&A, 23, 454

Yusef-Zadeh, F., \& Mehringer, D. M. 1995, ApJ, 452, L17 